\title{
Distant metastasis in retroperitoneal dedifferentiated liposarcoma is rare and rapidly fatal: a clinicopathological study with emphasis on the low-grade myxofibrosarcoma-like pattern as an early sign of dedifferentiation
}

\author{
Hsuan-Ying Huang ${ }^{1}$, Murray F Brennan ${ }^{2}$, Samuel Singer ${ }^{2}$ and Cristina R Antonescu ${ }^{1}$ \\ ${ }^{1}$ Department of Pathology, Memorial Sloan-Kettering Cancer Center, NY, USA and ${ }^{2}$ Department of Surgery, \\ Memorial Sloan-Kettering Cancer Center, NY, USA
}

\begin{abstract}
The metastatic incidence of retroperitoneal dedifferentiated liposarcoma is comparatively lower than other pleomorphic sarcomas, varying widely from 1 to $18 \%$. Low-grade dedifferentiation resembling low-grade fibrosarcoma has been recently accepted as part of the morphologic spectrum of dedifferentiated liposarcoma and was reported to have similar metastatic and survival rates to its high-grade counterpart. We sought to determine the metastatic incidence of retroperitoneal dedifferentiated liposarcoma, the clinicopathological features related to metastasis, and their postmetastatic behavior. Of all 354 retroperitoneal liposarcoma cases diagnosed at Memorial Sloan-Kettering Cancer Center during 1982-2003, we identified seven patients developing distant metastases, occurring in four females and three males, ranging from 35 to 73 years in age at presentation. They were all de novo dedifferentiated, while none of the well-differentiated liposarcoma or secondary dedifferentiated liposarcoma developed distant metastasis. Primary tumor sizes varied from 7.5 to $25 \mathrm{~cm}$. All seven patients developing metastases contained $\geq 50 \%$ dedifferentiated elements in the primary tumor, with a predominant morphology resembling myxofibrosarcoma in five cases. The metastatic sites included the lung in four patients, somatic soft tissue in two, and liver in one. The median metastasis-free survival was $\mathbf{4 8}$ months, with only two patients experiencing local recurrences before developing metastasis. Six patients died of disease at median follow-up of $\mathbf{5 3}$ months after diagnosis and only $\mathbf{5}$ months after their first metastases. In conclusion, retroperitoneal dedifferentiated liposarcoma have a low metastatic rate, which is strongly related to de novo dedifferentiated histology that usually constitutes a prominent component of the primary tumor. Irrespective of the grade dedifferentiated liposarcoma with myxofibrosarcoma-like features should be closely monitored. Once metastases occur, they tend to follow a rapidly fatal course.
\end{abstract} Modern Pathology (2005) 18, 976-984. doi:10.1038/modpathol.3800381; Published online 8 April 2005

Keywords: retroperitoneal; dedifferentiated liposarcoma; metastasis; myxofibrosarcoma-like; low-grade dedifferentiation

Dedifferentiated liposarcoma originally defined by Evans $^{1}$ in 1979 , consists of well-differentiated

Correspondence: Dr CR Antonescu, MD, Department of Pathology, Memorial Sloan-Kettering Cancer Center, 1275 York Avenue, New York, NY 10021, USA.

E-mail: antonesc@mskcc.org

This study was performed while Dr Hsuan-Ying Huang worked as a visiting research fellow in the Department of Pathology at Memorial Sloan-Kettering Cancer Center. His present address is Department of Pathology, Chang Gung Memorial Hospital, Kaohsiung, Taiwan.

Received 4 October 2004; revised and accepted 13 December 2004; published online 8 April 2005 liposarcomas associated with or progressing to a high-grade nonlipogenic sarcoma. ${ }^{1}$ Despite a 5 -year disease-specific mortality up to $30 \%$ for retroperitoneal dedifferentiated liposarcoma, the majority of fatal cases were caused by locally aggressive disease. $^{2,3}$ In spite of its commonly pleomorphic high-grade morphology, several clinicopathological studies have indicated that dedifferentiated liposarcoma behaves less aggressively as compared with other pleomorphic sarcomas of adulthood. ${ }^{4,5}$ Some authors considered that the basis for this difference might be related to a comparatively lower metastatic rate, ranging from 1 to $18 \%$ depending on series. ${ }^{1-7}$ 
Nevertheless, two points remained to be cautiously elucidated before drawing such a conclusion. Firstly, most patients with dedifferentiated liposarcoma die of the local effects of their tumor before metastasis become obvious; and secondly, it is difficult to accurately determine what criteria distinguish between local (contiguous) invasion and true metastasis.

Dedifferentiated areas in liposarcoma can display a variety of histological patterns, including pleomorphic/storiform malignant fibrous histiocytoma-like, ${ }^{1-6}$ fibrosarcoma-like,${ }^{1-6}$ myxofibrosarcoma-like,,$^{2,4,5,7}$ and heterologus differentiation, such as myogenic ${ }^{8,9}$ or osteogenic or chondro-sarcomatous ${ }^{8}$ elements, etc. In addition, the original concept of dedifferentiation in liposarcoma was recently challenged by Henricks et $a l^{2}{ }^{2}$ who expanded fibrosarcoma or fibromatosis-like appearances. As compared to the high-grade counterparts, low-grade dedifferentiation was reported to confer similar metastatic and survival rates.

Nevertheless, whether the current notion and hitherto described histological spectrum of dedifferentiation, including pure low-grade examples, are completely irrefutable necessitate reappraisal. For instance, although high-grade myxofibrosarcomalike appearance was widely accepted as a distinct morphological pattern of dedifferentiated liposarcoma, ${ }^{2,4,5,7}$ little is known about the prognostic significance and metastatic potential of low-grade myxofibrosarcoma-like histology in retroperitoneal dedifferentiated liposarcoma. In addition, the distinction between dedifferentiated liposarcoma showing heterologus components, ${ }^{8,9}$ such as osteosarcoma-like morphology, ${ }^{8}$ and malignant mesenchymoma characterized by the coexistence of well-differentiated liposarcoma and osteosarcoma still remains obscure. ${ }^{10-12}$ Therefore, the aim of current series was to better address the aforementioned questions by correlating the morphological variations, extent, and histological grades of dedifferentiation with the clinical evolution, preferential metastatic sites, and postmetastatic survival of seven patients developing distant metastasis from their retroperitoneal dedifferentiated liposarcoma.

\section{Materials and methods}

The prospectively maintained sarcoma database of Memorial Sloan-Kettering Cancer Center (MSKCC) was searched for retroperitoneal liposarcomas diagnosed between 1982 and 2003. After histological reclassification by excluding retroperitoneal pleomorphic $(n=15)$ and myxoid/round cell liposarcomas $(n=30)$, we found that 354 patients had primary and/or recurrent tumors belonging to the family of well-differentiated/dedifferentiated liposarcoma. Of these, primary de novo and secondary dedifferentiated liposarcoma were identified in $97(27 \%)$ and $31(9 \%)$, respectively. None of the patients had distant metastasis at the time of presentation. The seven patients developing distant metastases were included by recognizing bona fide parenchymal involvement of visceral organs or somatic soft tissues with discrete separation from the infiltrative borders of main tumors. Those tumors with local direct invasion/adherence to intra-abdominal organs, such as renal, splenic, or hepatic capsules, were not regarded as true distant spread. This metastatic subset represented $2 \%$ of all retroperitoneal well-differentiated/dedifferentiated liposarcomas, $5 \%$ of both primary and secondary retroperitoneal dedifferentiated liposarcoma, and $7 \%$ of primary retroperitoneal dedifferentiated liposarcoma, and their primary tumors were all de novo dedifferentiated.

In these seven patients, the following clinicopathological features were examined for the primary and/or locally recurrent sarcomas: age, sex, tumor size of primary tumor, the extent, histological grade, the predominant morphological patterns in both well-differentiated and dedifferentiated elements, mitotic rates, and percentage of tumor necrosis. The dedifferentiated component was graded as low or high, based on a two-tier grading scheme traditionally applied at MSKCC. A low-grade sarcoma was defined as a tumor with low cellularity, increased extracellular stroma, a mitotic rate $<5 \mathrm{MF} / 10 \mathrm{HPFs}$, and only focal tumor necrosis. The features of a high-grade lesion included increased cellularity with associated less extracellular stroma, mitotic activity of $\geq 5 \mathrm{MF} / 10 \mathrm{HPFs}$, and tumor necrosis of $10 \%$. For the metastatic lesions, the sites, histological features, and tumor grades were obtained by both histological and chart reviews. The time intervals to each local recurrence and distant metastasis, the last follow-up status, and duration of postmetastatic survival were compared with clinicopathological data.

\section{Results}

\section{Clinical Findings and Follow-Up}

The clinical features of the seven patients developing metastasis from the retroperitoneal dedifferentiated liposarcoma are summarized in Table 1. The patients comprised four women and three men, who ranged in age from 35 to 73 (mean, 59; median, 60) years. Except for abdominal fullness noted in two patients (case no. 1,5), there was no characteristic presenting complaint in the other patients. Surgical excision was the treatment for the primary tumors in all seven cases. The most common site of first metastasis was the lung/pleura $(n=4)$, followed by somatic soft tissue (one each for thigh and axilla), and liver $(n=1)$ after a median follow-up of 48 months (range, 4-144 months). One patient (case no. 5) experienced three episodes of local recurrences at 3 , 41, and 46 months after excision of the primary tumor and eventually developed metastasis to 
Table 1 Clinical features and follow-up data of seven retroperitoneal primary dedifferentiated liposarcomas developing distant metastases

\begin{tabular}{|c|c|c|c|c|c|c|c|c|}
\hline Case & $\begin{array}{c}\text { Age } \\
\text { (years) }\end{array}$ & Sex & $\begin{array}{c}\text { Size of } \\
\text { primary } \\
\text { tumor }(\mathrm{cm})\end{array}$ & Treatment & $\begin{array}{l}\text { Site of } \\
\text { first } \\
\text { metastasis }\end{array}$ & $\begin{array}{l}\text { Site of } \\
\text { subsequent } \\
\text { metastases }\end{array}$ & Follow-up & Postmetastasis survival \\
\hline 1 & 58 & $\mathrm{~F}$ & 25 & Surgery & $\begin{array}{l}\text { Thigh/soft } \\
\text { tissue }\end{array}$ & $\begin{array}{l}\text { Lung, pericardium, } \\
\text { liver }\end{array}$ & $\mathrm{DM}$ at $4 \mathrm{mos}$; DOD at $10 \mathrm{mos}$ & $6 \mathrm{mos}$ \\
\hline 2 & 64 & $\mathrm{~F}$ & 11 & Surgery & Lung & Liver & DM at 6 mos; DOD at 8 mos & 2 mos \\
\hline 3 & 63 & $\mathrm{~F}$ & 7.5 & Surgery & Lung, pleura & None & $\mathrm{DM}$ at 144 mos; DOD at 192 mos & $48 \mathrm{mos}$ \\
\hline 4 & 59 & M & $10-20$ & Surgery & Lung & None & $\mathrm{DM}$ at $8 \mathrm{mos}$; DOD at 19 mos & $11 \mathrm{mos}$ \\
\hline 5 & 35 & $\mathrm{~F}$ & $10-20$ & Surgery & $\begin{array}{l}\text { Axilla/soft } \\
\text { tissue }\end{array}$ & None & $\begin{array}{l}\mathrm{LR} \text { at } 3,41,46 \mathrm{mos} ; \mathrm{DM} \text { at } \\
48 \mathrm{mos} ; \mathrm{DOD} \text { at } 53.5 \mathrm{mos}\end{array}$ & $5 \mathrm{mos}$ \\
\hline 6 & 60 & $\mathrm{M}$ & 9.5 & Surgery & Liver & Pancreas & DM at 97 mos; DOD at 99 mos & 2 mos \\
\hline 7 & 73 & $\mathrm{M}$ & 15 & Surgery & Lung & None & LR at 23,66 mos; DM at 119 mos & Recent metastatic event \\
\hline
\end{tabular}

DM, distant metastasis; LR, local recurrence; DOD; dead of disease; mos, months.

Table 2 Histological features of seven primary retroperitoneal dedifferentiated liposarcomas developing distant metastases

\begin{tabular}{|c|c|c|c|c|c|c|}
\hline Case & $\begin{array}{l}\text { Well- } \\
\text { differentiated } \\
\text { component }\end{array}$ & $\begin{array}{c}\text { Percentage } \\
\text { of well- } \\
\text { dedifferentiated } \\
\text { component }\end{array}$ & $\begin{array}{l}\text { Dedifferentiated } \\
\text { component }\end{array}$ & $\begin{array}{c}\text { Percentage of } \\
\text { dedifferentiated } \\
\text { component }\end{array}$ & $\begin{array}{l}\text { Percentage of } \\
\text { tumor necrosis } \\
\text { in primary } \\
\text { tumors }\end{array}$ & $\begin{array}{l}\text { Histological appearance } \\
\text { of metastatic lesions }\end{array}$ \\
\hline 1 & Sclerosing & 10 & $\begin{array}{l}\text { High-grade fibrosarcoma- } \\
\text { like }\end{array}$ & 80 & 20 & $\begin{array}{l}\text { Fibromatosis or solitary } \\
\text { fibrous tumor-like }\end{array}$ \\
\hline 2 & Lipoma-like & 20 & $\begin{array}{l}\text { High-grade } \\
\text { osteochondrosarcoma- } \\
\text { like }\end{array}$ & 65 & 15 & $\begin{array}{l}\text { Giant cell-rich } \\
\text { malignant fibrous } \\
\text { histiocytoma-like }\end{array}$ \\
\hline 3 & Sclerosing & 50 & $\begin{array}{l}\text { Low-grade } \\
\text { myxofibrosarcoma-like }\end{array}$ & 50 & 0 & $\begin{array}{l}\text { Low-grade } \\
\text { myxofibrosarcoma-like }\end{array}$ \\
\hline 4 & Lipoma-like & 20 & $\begin{array}{l}\text { Low-grade } \\
\text { myxofibrosarcoma-like }\end{array}$ & 70 & 10 & $\begin{array}{l}\text { Low-grade } \\
\text { myxofibrosarcoma-like }\end{array}$ \\
\hline 5 & Lipoma-like $^{\mathrm{a}}$ & $0^{\mathrm{a}}$ & $\begin{array}{l}\text { High-grade } \\
\text { myxofibrosarcoma-like }\end{array}$ & 100 & 30 & $\begin{array}{l}\text { High-grade } \\
\text { myxofibrosarcoma-like }\end{array}$ \\
\hline 6 & Sclerosing & 10 & $\begin{array}{l}\text { High-grade } \\
\text { myxofibrosarcoma-like }\end{array}$ & 60 & 40 & $\begin{array}{l}\text { High-grade } \\
\text { myxofibrosarcoma-like }\end{array}$ \\
\hline 7 & Sclerosing & 30 & $\begin{array}{l}\text { Low-grade } \\
\text { myxofibrosarcoma-like }\end{array}$ & 50 & 10 & $\begin{array}{l}\text { High-grade } \\
\text { myxofibrosarcoma-like }\end{array}$ \\
\hline
\end{tabular}

\footnotetext{
${ }^{\mathrm{a}}$ Lipoma-like well-differentiated component become apparent in the local recurrent tumor.

${ }^{\mathrm{b}}$ The myxofibrosarcoma-like area in this dedifferentiated liposarcoma progressed to a high-grade histology in the 1st and 2nd local recurrences before development of lung metastasis.
}

axillary soft tissue at 48 months. Another patient (case no. 7) had two local recurrences at 23 and 66 months, with subsequent multiple pulmonary metastases at 119 months. The remaining five patients displayed metastatic diseases in the absence of prior local recurrences. After the first metastasis, subsequent dissemination was also detected in the visceral organs of three patients, including the lung, liver, pancreas, and pericardium, as documented by radiological follow-up or autopsy reports. In the present series, six of seven patients died of their diseases with a median survival of 53 months (range, 8-192 months), which appeared to be comparatively worse than the entire cohort of retroperitoneal dedifferentiated liposarcoma. The basis of this remarkable difference in prognosis might be ascribed to the extremely aggressive postmetastatic course of metastatic retroperitoneal dedifferentiated liposarcoma with a median postmetastatic survival of only 5 months.

\section{Pathological Findings}

Grossly, the primary tumors were described as tanpink or yellow with soft (case no. 3-7), rubbery (case no. 1), to focally firm gritty (case no. 2) consistency. The tumor size ranged from 7.5 to $25 \mathrm{~cm}$. Distinct gelatinous, single nodular or multinodular areas were present in five cases (case no. 3-7). The histological features of these seven cases are listed in Table 2. All seven primary tumors were de novo 
dedifferentiated. The metastatic lesions displayed a pure dedifferentiated morphology without a well-differentiated component. The well-differentiated component was observed in the primary lesions of six cases, accounting for $10-50 \%$ of surface areas examined. In the remaining case it became apparent in the first local recurrence (case no. 5). The predominant histology of the welldifferentiated areas included three lipoma-like and four sclerosing histological subtypes. The former was composed mostly of a lobular proliferation of fat cells characterized by marked variation in size and shape, as well as variable amounts of mono- or multivacuolated lipoblasts with scalloping nuclei. The sclerosing subtype contained areas of dense fibrocollagenous matrix harboring scattered atypical stromal cells, floret-like giant cells, and fat cells, including lipoblasts.

The common but striking similarity in all seven primary tumors was that dedifferentiated areas formed the predominant component $(\geq 50 \%$ of the mass; mean, $67 \%$ ). In case no. 1 , the primary tumor showed an abrupt transition between the paucicellular, sclerosing well-differentiated and the high-grade fibrosarcoma morphology. The latter component displayed either a fascicular growth or resembled a malignant fibrous solitary tumor pattern, with interspersed sunburst-shaped amianthoid collagen fibers and staghorn vasculature (Figure 1a and b). The mitotic count was $8 \mathrm{MF} / 10 \mathrm{HPF}$ in the most mitotically active area of primary tumor, and coagulative tumor necrosis was focally seen. Nevertheless, the first metastasis to the right thigh displayed a relatively circumscribed, hypocellular, fibroblastic appearance characterized by abundant collagen fibers, thereby resembling a benign solitaryfibrous tumor or fibromatosis without obvious mitoses or tumor necrosis (Figure 1c).

In case no. 2, the well-differentiated component was of lipoma-like subtype, while the adjacent pleomorphic sarcomatous zone was hypercellular with discernible tumor necrosis and a mitotic rate up to $12 \mathrm{MF} / 10 \mathrm{HPF}$. This latter component featured heterologus differentiation showing both hyaline chondroid matrix and intricate mineralizing osteoid (Figure 2a-c). In addition, scattered collections of osteoclast-like giant cells were found admixed with sarcomatous cells in the osteoid-producing area of the primary tumor (Figure 2d). The solitary lung metastasis was rich in osteoclast-like giant cells but devoid of matrix, thereby simulating a giant cell malignant fibrous histiocytoma (Figure 2e).

The common denominator in the dedifferentiated portion of the remaining five cases was the predominant myxofibrosarcoma-like appearance. Nodular myxoid proliferations distinct from the adjacent well-differentiated liposarcoma elements were identified in four primary lesions, but present only in the local recurrences in the remaining one (Figures 3a, 4a and b) (see Table 2). The myxofibrosarcoma-like dedifferentiated areas contained spindle, stellate, or
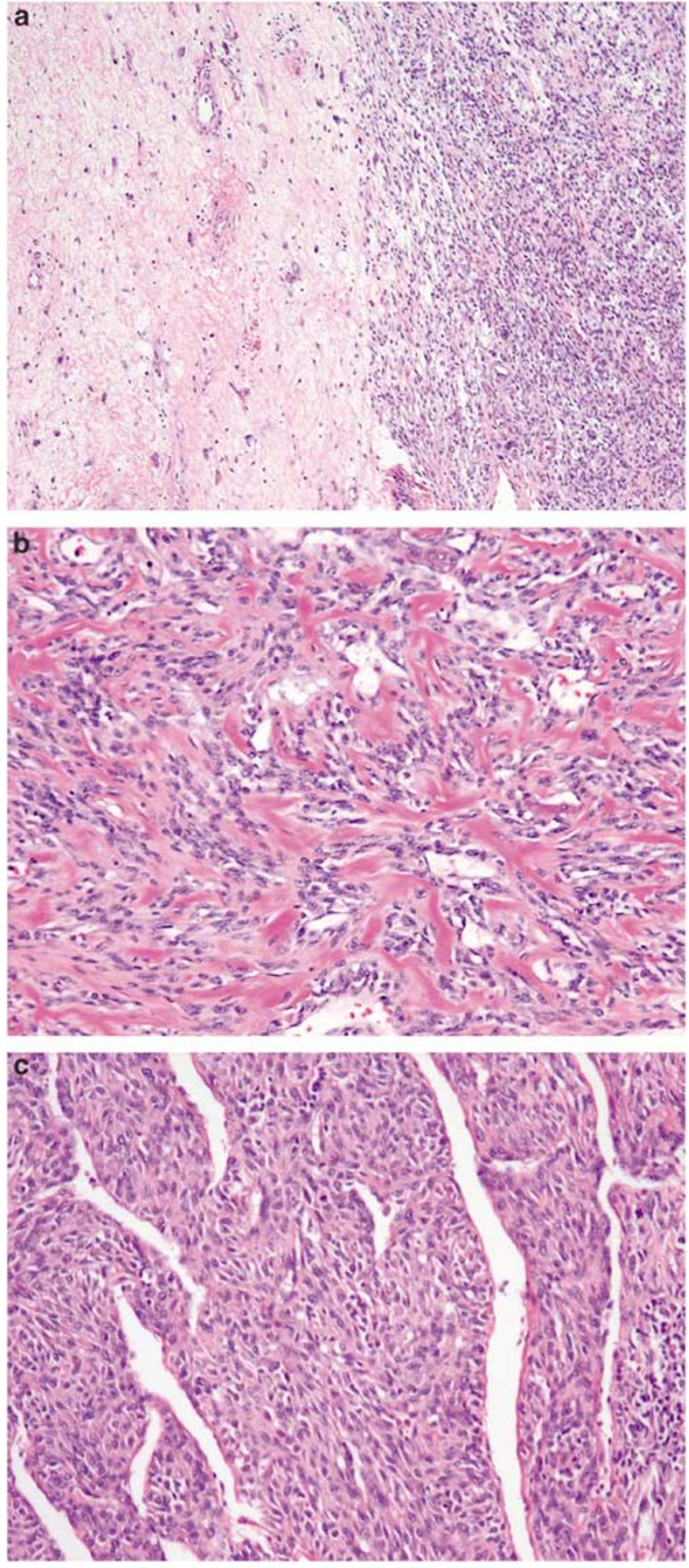

Figure 1 (a) The primary tumor of case 1 showed an abrupt transition between the components of well-differentiated sclerosing liposarcoma and high-grade fibrosarcoma-like dedifferentiated liposarcoma (H\&E, $\times 40)$. (b) In other area of dedifferentiated component of the same tumor, a solitary fibrous tumor-like pattern characterized by interspersed amianthoid collagen fibers of sunburst configuration was seen $(\mathrm{H} \& \mathrm{E}, \times 100)$. (c) This case developed a distant metastasis to the soft tissue of thigh, which showed a solitary-fibrous tumor-like histology (H\&E, $\times 100)$.

bizarre cells of varying degree of cellularity and pleomorphism, embedded in a prominent myxoid stroma rich in thick curvilinear vasculature or finer 

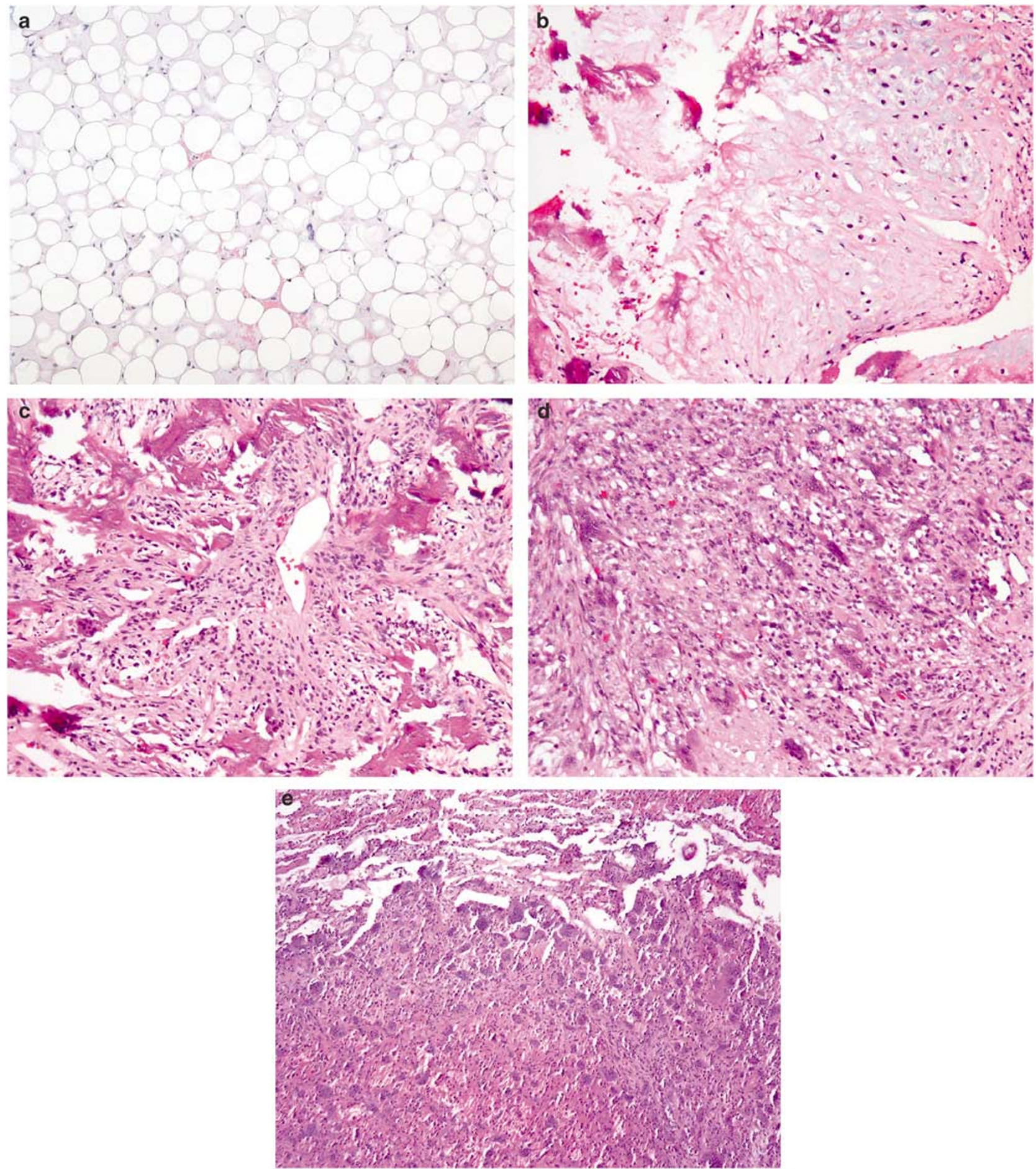

Figure 2 The primary tumor of case 2 showed the components of well-differentiated lipoma-like liposarcoma (a, H\&E, $\times 100)$ and heterologus dedifferentiated liposarcoma displaying chondrosarcoma-like $(\mathbf{b}, \mathrm{H} \& \mathrm{E}, \times 100)$ and osteosarcoma-like $(\mathbf{c}, \mathrm{H} \& \mathrm{E}, \times 100)$ histological patterns with focal collection of many osteoclast-like giant cells $(\mathbf{d}, \mathrm{H} \& \mathrm{E}, \times 100)$. The pulmonary metastasis of this case revealed a giant cell-rich tumor nodule $(\mathbf{e}, \mathrm{H} \& \mathrm{E}, \times 40)$ similar to the morphological features of $\mathbf{d}$.

plexiform capillary network (Figures 3a, 4a and b). The histologic grade of the dedifferentiated component in the primary lesions was low grade (Figure $3 a$ ) in three and high grade (Figure $4 \mathrm{~b}$ ) in two cases, using a two-tier grading scheme. The low-grade group was defined as having low cellularity, slight nuclear pleomorphism and low mitotic rates $<5$ / $10 \mathrm{HPF}$. The high-grade group had increased cellularity, moderate to marked nuclear pleomorphism, mitotic activity of $\geq 5 / 10 \mathrm{HPFs}$, and tumor necrosis. 

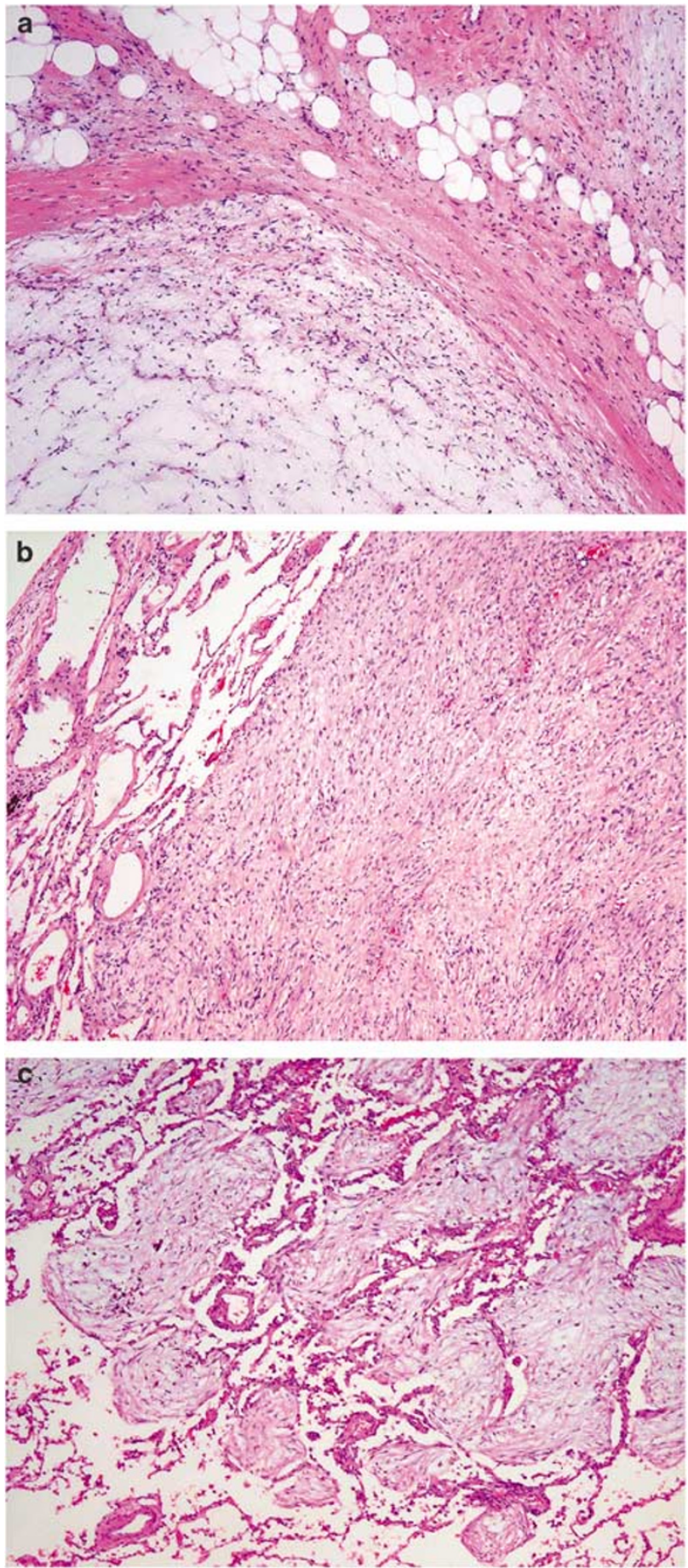

Figure 3 (a) The primary tumor of case 3 revealed the interface between the components of well-differentiated sclerosing liposarcoma (upper right) and low-grade myxofibrosarcoma-like dedifferentiated liposarcoma (left lower) (H\&E, $\times 40)$; the latter was characterized by myxoid nodular appearance, curvilinear vessels, and hypocellular proliferation of bland spindly to stellate mesenchymal cells set in the abundant myxoid matrix. (b) The same case developed multiple nodular metastases to the lung with less myxoid stroma but retained the identical histological grade $(\mathrm{H} \& \mathrm{E}, \times 40)$. (c) The lung metastasis from another RPDLS with low-grade myxofibrosarcoma-like dedifferentiated pattern (cases 4) displayed a peculiar 'BOOP-like' appearance with many intraalveolar and/or peribronchiolar tumor micronodules composed of bland tumor cells within abundant myxoid matrix $(\mathrm{H} \& \mathrm{E}, \times 100)$.

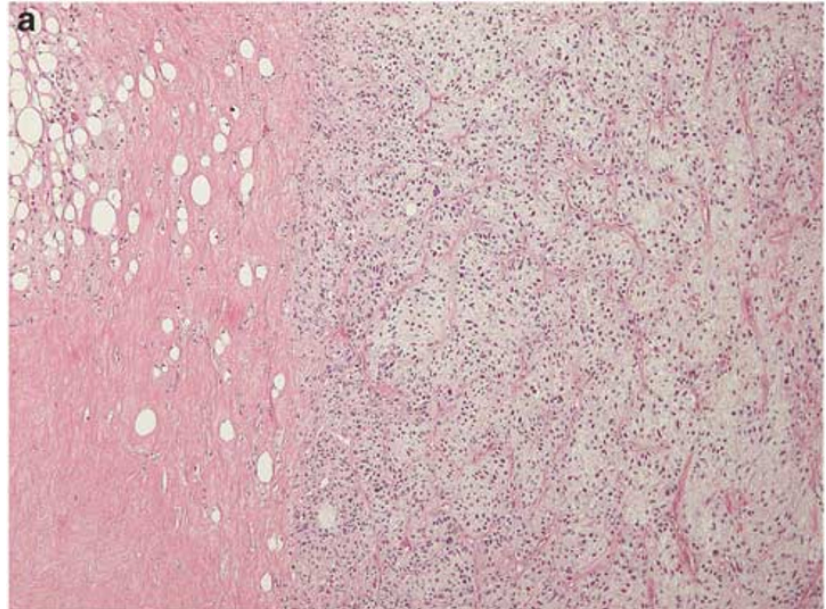

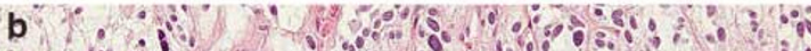

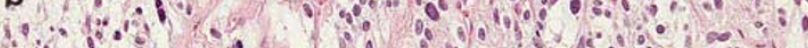

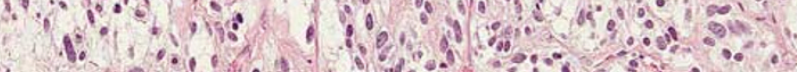

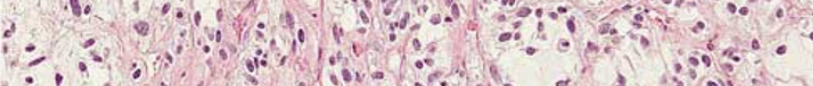

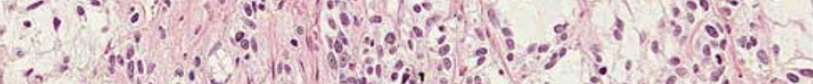

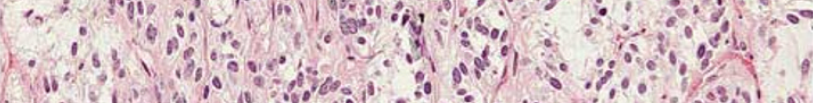

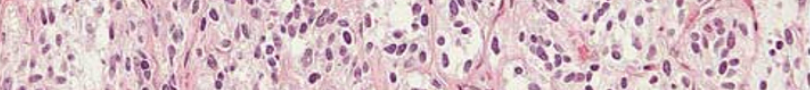

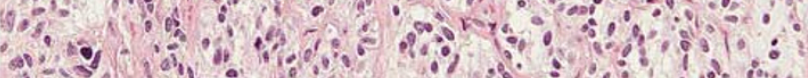
a

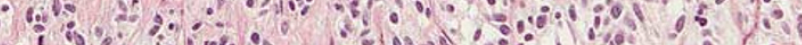

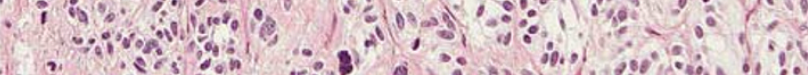

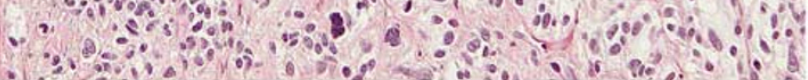

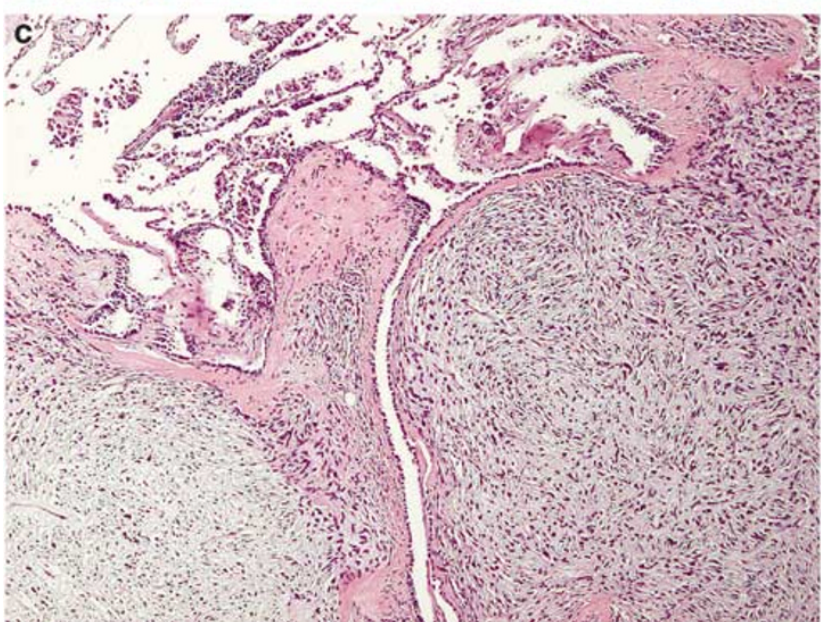

Figure 4 The recurrent tumor of case 7 showed a welldifferentiated sclerosing liposarcoma adjacent to the high-grade myxofibrosarcoma-like dedifferentiated component (a, H\&E, $\times 40$ ) showing scattered tumor giant cells and curvilinear vessels in the myxoid matrix $(\mathbf{b}, \mathrm{H} \& \mathrm{E}, \times 100)$. The same case developed pulmonary metastatic lesions with high-grade myxofibrosarcomalike appearance with moderate cellularity and marked nuclear pleomorphism $(\mathbf{c}, \mathrm{H} \& \mathrm{E}, \times 100)$.

The metastatic lesions generally retained the same histological grade as those of the primary lesions, and displayed either similar myxoid or somewhat 
more fibrous stroma (Figure $3 \mathrm{~b}$ and c). However, the two lung metastases of case no. 7 were both high grade (Figure 4c), identical to the prior local recurrence, progressing from a primary tumor with low-grade dedifferentiation. Of note were the peculiar 'BOOP-like' intra-alveolar or peribronchiolar micronodular patterns seen in some of the metastases of case no. 3 (Figure 3c).

\section{Discussion}

Well-differentiated and dedifferentiated liposarcoma, the most common retroperitoneal sarcoma, are considered to lie within the same spectrum, as dedifferentiated liposarcoma represents a manifestation of disease progression from well-differentiated component in the primary tumors or recurrences. ${ }^{1,2,6}$ Dedifferentiated liposarcoma typically displays a variable histological picture, but most frequently resembles malignant fibrous histiocytoma or intermediate to high-grade myxofibrosarcoma. ${ }^{1,2,4-7}$ Careful and extensive sampling is therefore mandatory, particularly in primary $d e$ novo dedifferentiated liposarcoma with prominent dedifferentiation, as the well-differentiated component may be neglected. Both well-differentiated and dedifferentiated liposarcoma are characterized cytogenetically by ring or giant marker chromosomes derived from amplified 12q13-15 material, with additional aberrations more often detected in dedifferentiated variants. ${ }^{13-15}$ Indeed, it was demonstrated recently that a subgroup of previously diagnosed malignant fibrous histiocytoma, especially for those arising in the retroperitoneum, is genetically similar with well-differentiated/dedifferentiated liposarcoma family. ${ }^{13,14}$

Although dedifferentiation is associated with a six-fold increased risk of death as compared with well-differentiated histology, ${ }^{16}$ some authors believe that dedifferentiated liposarcoma has a low incidence of distant metastasis, ranging from 1 to $18 \%$ in various series. ${ }^{2-9}$ Other pleomorphic sarcomas, such as storiform/pleomorphic malignant fibrous histiocytoma of extremities may show a cumulative metastatic probability greater than $50 \%$ at 3 years. ${ }^{16,17}$ The present series substantiated this viewpoint by finding that only $7 \%$ of 97 primary retroperitoneal dedifferentiated liposarcomas, followed in a prospective database of MSKCC and treated in a standard fashion, eventually developed distant metastasis proven by histological diagnosis. Nevertheless, it must be added that this figure may represent a minimum metastatic rate since we adopted strict histological criteria to define bona fide metastasis.

Henricks et $a l^{2}$ investigated the impact of the extent, grading, and histological pattern of dedifferentiation on outcome. In their analysis of 155 dedifferentiated liposarcomas, including 106 retroperitoneal cases, none of the histological factors studied proved predictive of patient survival and metastatic propensity. ${ }^{2}$ Their cases of dedifferentiated liposarcomas with pure low-grade fibrosarcoma/fibromatosis-like appearance even showed a slightly higher (25 vs 16\%), albeit insignificant, incidence of metastases. ${ }^{2}$ We found that primary de novo dedifferentiated liposarcoma had an increased risk of distant metastasis compared with secondary dedifferentiation, insofar as all seven patients developing metastases in this study had $a b$ initio dedifferentiation. Two possible causes may account for this result. Firstly, de novo lesions are more advanced temporally in view of tumor progression, since they have already progressed from welldifferentiated areas for a period of time. Secondly, the prominent rather than focal dedifferentiated component seen in our cases is more likely to harbor subclones of sarcoma cells with metastatic capability. To date, no investigation specifically addressed the postmetastatic course in patients with metastatic dedifferentiated liposarcoma and little is known about whether low-grade dedifferentiation differs from high-grade counterpart in this regard. This question is relevant given patients with other metastatic low-grade sarcomas, such as low-grade fibromyxoid sarcoma ${ }^{18}$ and endometrial stromal sarcoma, ${ }^{19}$ that usually have a prolonged survival if the metastases are surgically excised. In the current series, we found that the postmetastatic course was extremely aggressive, since all patients died of systemic dissemination of tumors at a median of 5 months after developing first metastases, except for case no. 7 having a recent metastatic event. In addition, this finding appears to be independent of histological grade, since the two metastatic cases with low-grade myxofibrosarcoma-like histology (case no. 3,4 ) both died of their diseases, with postmetastatic survival time comparable to the high-grade tumors.

Myxofibrosarcoma-like histology in retroperitoneal liposarcoma represents a distinct type of dedifferentiation with a broad spectrum of tumor progression analogous to soft tissue myxofibrosarcoma. ${ }^{20}$ These can range from a very bland, low-grade pattern almost indistinguishable from well-differentiated liposarcoma with secondary myxoid change to frankly high-grade dedifferentiation with gradually increased mitotic activity and nuclear pleomorphism. ${ }^{7,21}$ Overall, myxofibrosarcoma-like histology (five out of seven, $71 \%$ ) constituted the most common subtype of dedifferentiation in this series, implying a special intrinsic propensity of myxoid matrix for the development of metastasis. Further characterization of these findings may provide insight into the biology of these tumors. The recognition of low-grade myxofibrosarcoma-like histology as a novel concept of dedifferentiation with metastatic propensity was validated in two of our patients (case no. 3, 4) showing the same low-grade appearance in metastatic lesions. Nevertheless, its morphological distinction from well- 
differentiated liposarcoma with myxoid change is admittedly difficult at times without standardized guidelines. Based on histological review of all retroperitoneal liposarcomas with myxoid areas at our institution, we proposed that tumors should be identified as having low-grade myxofibrosarcoma-like dedifferentiation, provided that (1) lack adipocytic differentiation in at least one low-power view area; (2) are associated with a prominent vascular networks, either chicken-wire capillary or thicker curvilinear in shape within the myxoid nodular areas; and (3) show presence of increased cellularity with at least mild nuclear atypia.

Approximately one-third of retroperitoneal dedifferentiated liposarcomas contain low and/or high-grade myxofibrosarcoma-like areas, ${ }^{7,21}$ which may pose a tremendous diagnostic challenge due to overlapping morphological features with other myxoid neoplasms. This difficulty is especially obvious in the distinction from myxoid liposarcoma and myxofibrosarcoma, ${ }^{7,21,22}$ and generate speculation upon the existence of true mixed liposarcoma in this location. According to our reclassification stated in Materials and methods, bona fide myxoid/ round cell liposarcomas accounted for only $7 \%$ of all retroperitoneal liposarcomas, some of which were previously verified by the presence of the hallmark TLS-CHOP fusion gene, ${ }^{21,22}$ suggesting the rarity but actual existence of this entity in the retroperitoneum. On the other hand, in our prior series we failed to detect the chimeric fusion transcript of TLS-CHOP or CHOP gene rearrangement by using reverse transcriptase polymerase chain reaction or Southern blot assay in any of well-differentiated or dedifferentiated liposarcomas, with either predominantly (defined as myxoid area $>90 \%$ ) or less myxoid histology (myxoid area $\leq 50 \%$ ). ${ }^{21}$ These molecular results suggested that dedifferentiated liposarcoma with myxofibrosarcoma-like morphology is histogenetically distinct from the translocation-associated myxoid/round cell liposarcoma. In addition, adequate sampling is mandatory to distinguish among true myxofibrosarcoma, ${ }^{22}$ myxofibrosarcoma-like pleomorphic liposarcoma, ${ }^{23}$ and myxofibrosarcoma-like dedifferentiated liposarcoma ${ }^{7,21}$ of the retroperitoneum. Rendering definite diagnoses for the latter two entities depend on the identification of telltale welldifferentiated liposarcoma component (lipoma-like or sclerosing) $)^{7,21}$ and pleomorphic lipoblasts. ${ }^{23}$ The distinction between dedifferentiated liposarcoma with myxofibrosarcoma-like histology and other subtypes of myxoid sarcomas is crucial in predicting the biological behavior. While myxoid liposarcoma has a greater propensity for somatic soft tissue metastasis as compared to other histological type of sarcomas, ${ }^{24}$ this property is not restricted to myxoid liposarcoma alone. In the present report, one each of high-grade fibrosarcoma- and myxofibrosarcoma-like dedifferentiated liposarcoma (case no. 1, 5) developed distant metastases firstly to soft tissue sites. Apart from the common pulmonary and osseous metastases, lymph node metastases are seen in a small but significant number of myxofibrosarcoma cases. ${ }^{22,25}$

The term 'malignant mesenchymoma' has been applied to sarcomas that exhibited two or more lines of specialized differentiation. ${ }^{10-12}$ In spite of strict diagnostic criteria, the existence of malignant mesenchymoma remains controversial and in the latest WHO classification on soft tissue tumors was not regarded as a distinct clinicopathologic entity. ${ }^{26}$ Dedifferentiated liposarcoma may exhibit heterologous differentiation in about $5 \%$ of cases, mainly with osteo-/chondro-sarcoma or myosarcomatous elements. ${ }^{8,9}$ One report of malignant mesenchymoma showing well-differentiated liposarcoma combined with divergent osteogenic sarcoma or myosarcoma contained ring chromosomes as well as amplification of $12 \mathrm{q} 13-\mathrm{q} 14 .^{12}$ These preliminary data seem to suggest that a subset of malignant mesenchymoma with well-differentiated liposarcoma as one of lines of differentiation might be histogenetically identical to dedifferentiated liposarcoma with heterologous elements.

In summary, we have described seven patients developing distant metastases from their retroperitoneal dedifferentiated liposarcoma. The tumors contained $50 \%$ or more of de novo dedifferentiated component in their primary lesions. Interestingly, not all patients experienced local recurrence, as seen in five cases of this series, before developing distant metastases. Although the number of cases in our study is relatively small, this metastatic subset appeared to display a shorter median survival than the entire cohort of dedifferentiated liposarcoma, primarily ascribed to an extremely aggressive postmetastatic course. Our findings support the notion of low-grade dedifferentiation and expand its morphological spectrum by virtue of the following reasons. First, despite limited case number, myxofibrosarcoma-like dedifferentiation appears to be the most common morphology prone to develop metastasis. Second, the myxofibrosarcoma-like pattern appears to be a continuum of tumor progression, with its low-grade end representing early dedifferentiation already carrying a metastatic potential. Finally, retroperitoneal dedifferentiated liposarcoma with low-grade myxofibrosarcoma-like dedifferentiation should not be confused with myxoid liposarcoma, a distinct subtype of liposarcoma, rarely arising primarily in the retroperitoneum, in light of their similarities in bland myxoid morphology and tendency to soft tissue metastasis.

\section{Acknowledgements}

We want to thank Milagros Lugo for editorial assistance and Monica Cha and Allison Samaniego for excellent support in providing clinical information from the MSKCC sarcoma database. 


\section{References}

1 Evans HL. Liposarcoma: a study of 55 cases with a reassessment of its classification. Am J Surg Pathol 1979;3:507-523.

2 Henricks W, Chu Y, Goldblum JR, et al. Dedifferentiated liposarcoma: a clinicopathological analysis of 155 cases with a proposal for an expanded definition of dedifferentiation. Am J Surg Pathol 1992;11: 1051-1058.

3 Weiss SW, Rao VK. Well-differentiated liposarcoma (atypical lipoma) of deep soft tissue of the extremities, retroperitoneum, and miscellaneous sites. A follow-up study of 92 cases with analysis of the incidence of 'dedifferentiation'. Am J Surg Pathol 1992;16: 1051-1058.

4 McCormick D, Mentzel T, Beham A, et al. Dedifferentiated liposarcoma. Clinicopathologic analysis of 32 cases suggesting a better prognostic subgroup among pleomorphic sarcomas. Am J Surg Pathol 1994;18: 1213-1223.

5 Hasegawa T, Seki K, Hasegawa F, et al. Dedifferentiated liposarcoma of retroperitoneum and mesentery: varied growth patterns and histological grades-a clinicopathologic study of 32 cases. Hum Pathol 2000;31: 717-727.

6 Elgar F, Goldblum JR. Well-differentiated liposarcoma of the retroperitoneum: a clinicopathologic analysis of 20 cases, with particular attention to the extent of lowgrade dedifferentiation. Mod Pathol 1997;10:113-120.

7 Hisaoka M, Morimitsu Y, Hashimoto H, et al. Retroperitoneal liposarcoma with combined well-differentiated and myxoid malignant fibrous histiocytoma-like myxoid areas. Am J Surg Pathol 1999;23:1480-1492.

8 Evans HL, Khurana KK, Kemp BL, et al. Heterologous elements in the dedifferentiated component of dedifferentiated liposarcoma. Am J Surg Pathol 1994;18: 1150-1157.

9 Tallini G, Erlandson RA, Brennan MF, et al. Divergent myosarcomatous differentiation in retroperitoneal liposarcoma. Am J Surg Pathol 1993;17:546-556.

10 Newman PL, Fletcher CD. Malignant mesenchymoma. Clinicopathologic analysis of a series with evidence of low-grade behaviour. Am J Surg Pathol 1991;15: 607-614.

11 Brady MS, Perino G, Tallini G, et al. Malignant mesenchymoma. Cancer 1996;77:467-473.

12 Geurts van Kessel A, Simons A, Comtesse PP, et al. Ring chromosomes in a malignant mesenchymoma. Cancer Genet Cytogenet 1999;109:119-122.

13 Chibon F, Mariani O, Derre J, et al. A subgroup of malignant fibrous histiocytomas is associated with genetic changes similar to those of well-differentiated liposarcomas. Cancer Genet Cytogenet 2002;139: $24-29$.
14 Coindre JM, Mariani O, Chibon F, et al. Most malignant fibrous histiocytomas developed in the retroperitoneum are dedifferentiated liposarcomas: a review of 25 cases initially diagnosed as malignant fibrous histiocytoma. Mod Pathol 2003;16:256-262.

15 Forus A, Larramendy ML, Meza-Zepeda LA, et al. Dedifferentiation of a well-differentiated liposarcoma to a highly malignant metastatic osteosarcoma: amplification of $12 \mathrm{q} 14$ at all stages and gain of 1q22-q24 associated with metastases. Cancer Genet Cytogenet 2001;125:100-111.

16 Singer S, Antonescu CR, Riedel E, et al. Histologic subtype and margin of resection predict pattern of recurrence and survival for retroperitoneal liposarcoma. Ann Surg 2003;238:358-370.

17 Salo JC, Lewis JJ, Woodruff JM, et al. Malignant fibrous histiocytoma of the extremity. Cancer 1999;85:17651772.

18 Evans HL. Low-grade fibromyxoid sarcoma. A report of 12 cases. Am J Surg Pathol 1993;17:595-600.

19 Aubry MC, Myers JL, Colby TV, et al. Endometrial stromal sarcoma metastatic to the lung: a detailed analysis of 16 patients. Am J Surg Pathol 2002;26:440-449.

20 Huang H, Lal P, Qin J, et al. Clinicopathological and survival analysis of 49 myxofibrosarcomas at the low grade end of the spectrum-with simultaneous assessment of the efficacy of 3-tier and 4-tier grading systems. Hum Pathol 2004;35:612-621.

21 Antonescu CR, Elahi A, Humphrey M, et al. Specificity of TLS-CHOP rearrangement for classic myxoid/round cell liposarcoma: absence in predominantly myxoid well-differentiated liposarcomas. J Mol Diagn 2000;2: 132-138.

22 Antonescu CR, Tschernyavsky SJ, Decuseara R, et al. Prognostic impact of P53 status, TLS-CHOP fusion transcript structure, and histological grade in myxoid liposarcoma: a molecular and clinicopathologic study of 82 cases. Clin Cancer Res 2001;7:3977-3987.

23 Gebhard S, Coindre JM, Michels JJ, et al. Pleomorphic liposarcoma: clinicopathologic, immunohistochemical, and follow-up analysis of 63 cases: a study from the French Federation of Cancer Centers Sarcoma Group. Am J Surg Pathol 2002;26:601-616.

24 Spillane AJ, Fisher C, Thomas JM. Myxoid liposarcoma-the frequency and the natural history of nonpulmonary soft tissue metastases. Ann Surg Oncol 1999;6:389-394.

25 Mentzel T, Calonje E, Wadden C, et al. Myxofibrosarcoma. Clinicopathologic analysis of 75 cases with emphasis on the low-grade variant. Am J Surg Pathol 1996;20:391-405.

26 Fletcher CD, Umni K, Mertens F, et al. Malignant mesenchymoma. In: WHO Classification of Tumors: Pathology and Genetics of Tumors of the bone and soft tissue. IARC Press: Lyon, France, 2002, p 215. 